\section{Afferent Innervation of Mammalian Abdominal Viscera}

THE afferent nervous pathways from abdominal viscera have been studied by MeSwiney and his co-workers by means of the changes in pupil diameter induced by stimuli such as weak faradic shocks applied to the central end of the cut splanchnic nerve (Bain et $a l_{.}{ }^{1}$ ), or to the central end of the cut abdominal vagus nerve (Harper et $a l .{ }^{2}$ ). Investigations, by oscillographic methods, of the afferent nervous activity in the frog's viscera have been carried out by Tower ${ }^{3}$, and later confirmed by Burns ${ }^{4}$. It was shown that the gut and mesentery gave rise to fast nerve impulses in the sympathetic rami in response to light touch. These impulses. are not dissimilar to those in cutaneous sensory nerves in response to light touch on the frog's skin. On examining the afferent nervous mechanism in visceral nerves of the cat and rabbit, it has now been shown that a somewhat similar mechanism sensitive to light touch is present. Thus in response to light touch on the gut or the mesentery, trains of impulses of a fast type can be shown to ascend in the small nerve trunks which run, in company with blood vessels, across the mesentery from its intestinal attachment towards the mesenteric root. The exact distribution of this tactile sensibility and the function of the cat's mesenteric Paccinian corpuscles are being investigated.

Expenses grants towards this work from the Medical Research Council and the Government Grants Committee of the Royal Society are gratefully acknowledged.

Department of Physiology,

W. Burns. Marischal College, Aberdeen.

Jan. 26.

${ }^{1}$ Bain, W. A., Irving, J. T., and McSwiney, B. A., J. Physiol., 84, 323 (1935).

Harper, A. A., MeSwiney, B. A., and Suffolk, S. F., J. Physiol., 85. 267 (1935).

3 Tower, S. S., J. Physiol., 78, 225 (1933).

'Burns, W., J. Physiol., 100, 11P (1941).

\section{Biology and War}

IN these days when both comfort and efficiency are so dependent upon science and its applications, it is surely astonishing that so little weight is attached to the teachings of biological-apart from medical and agricultural-science in relation to problems which in these days have urgent importance.

An impressive example is afforded by the continued misuse of paint or pigment in relation to camouflage although the main principles of this were worked out more than a quarter of a century ago. Another is seen in the development of aircraft with refusal to grasp the fact that Nature had provided in the bodies of rapidly swimming fish a ready-made demonstration of the streamlined form most effective for rapid movement, whether through a denser medium such as water or a more tenuous one such as air. It is only now, after much needless expenditure of time and money, that the fuselage of fighter aircraft is seen to attain the correct form indicated by Nature.

Still another is concerned with the varying power of 'light' of different wave-lengths, as regards, on one hand, visibility by the human eye and, on the other, power of penetrating the atmosphere. A walk along a straight road on a hazy winter evening is enough to show by the reddening of the lamps with increasing distance the greater penetrating power of rays towards the red end of the spectrum. A walk by day when the sky is clear but the ground en. veloped in thick haze shows a complete absence of the sharp shadows of sunlight; and yet the photographer armed with films sensitive to infra-red rays sees these shadows sharply depicted in his photographs, the infra-red rays finding their way past the water particles suspended in the atmosphere until these become big enough to form wet fog or rain. Yet no steps are taken to compel all ships of considerable size to be equipped with the necessary apparatus - photographic or fluorescent-to see through haze dense enough to conceal dangers to navigation though fine enough in the size of its water particles to allow the passage of the infra-red rays.

Blindness to such simple examples is necessarily accompanied by blindness to more elusive teachings of biology. Perhaps the most striking feature in the evolution of animal organisms is their advancement in size from small microbes to the relatively large ereatures familiar in the animal world of to-day. This onward progress is a normal feature of evolution. It brings with it increased efficiency, increased power, increased speed of movement. But it also brings with it the seeds of decay and death. The geological record of evolution is littered with the remains of great powerful creatures which have become extinct while their smaller, feebler contemporaries have survived. The increase in size has in fact been attainable only by a corresponding increase in complexity of organization, involving the development of an ever greater multiplicity of parts, an ever greater sensitiveness to impressions from without, an ever greater centralization of control both over normal activities and over the appropriate reactions to emergencies. All this brings with it serious danger: vital processes are apt to become clogged and slowed down; destruction may come to the whole through the failure of some small part.

Blindness to such biological facts finds expression in many of our troubles of to-day. The air is full of complaints regarding the slowness of Government departments to act or to react-slowness which at times may have disastrous consequences. It is unfair in such cases to concentrate all the blame upon particular officials. It should rather be realized that many departmental shortcomings are inevitable results of their growth in size; and the only sure way to get rid of them is to diminish the patency of the size factor by a reversal of the normal evolutionary development. In some cases this can be effected by the resolution of the overgrown department into a number of more or less independent smaller ones. In others where this is not practicable, much can be done by the free delegation of authority by the central government to local bodies familiar with local conditions and free to take action on their own responsibility. The obvious dislike of the central departments to delegate any of their authority, to give up any of their powers of control, seems sometimes to imply a cynical disbelief in the reasonableness, or indeed the honesty, of the average citizen-a view which if it exists is most undoubtedly wrong.

The Athenæum,

Pall Mall,

Landon, S.W.1.

Feb. 4. 